\title{
The Design of Sea Water Aquaculture Water Quality Monitoring System Based on Zigbee and Data Fusion
}

\author{
Yujie Chen ${ }^{1, a}$, Peixue Liu ${ }^{1, b}$, Feng Chen ${ }^{1, c}$ and Qianqian $\mathrm{He}^{1, \mathrm{~d}}$ \\ ${ }^{1}$ School of QingDao HuangHai College, Qingdao 266427, China. \\ a79572611@qq.com; ${ }^{\text {b93937898@qq.com; }{ }^{c} 364367360 @ q q . c o m ;{ }^{d} 15964951785 @ q q . c o m}$
}

Keywords: Water Quality Monitoring, Zig Bee, data fusion, STM32F103ZET6

\begin{abstract}
Aiming at the current mariculture problem ofbackward environmental monitoring technology, design amonitoring system base on ARM processor STM32F103ZET6 and RF chip $\mathrm{CC} 2530$. Using wireless sensor nodes to collecttemperature, $\mathrm{pH}$ value, dissolved oxygen and other informationin Sea culture zone. Through the ARM processor, respectively, average the collect data and processing data based on adaptive weighted average algorithm, Send the processed data through the Zig Bee wireless, The coordinator access networks byGPRS, monitor LAN and remote computers with software platform.
\end{abstract}

\section{Introduction}

Mariculture is an important pillar of our coastal marine economy, The current monitoring methods mariculture,First,artificial way,this way less investment, poor accuracy, and timeliness is low; Second, wired control network monitoring methods, this approach requires a lot of on-site wiring, layout, maintenance and upgrade of operations difficult and costly. In view of this lack of modern technology and the status quo, this article is designed mariculture water quality monitoring system based on Zigbee and data fusion, In order to achieve real-time monitoring wireless remote, more comprehensive data collection, more efficient handling of gathering information, improve production efficiency mariculture.

\section{System Design}

System consists of a sensor terminal node, routing nodes, the coordinator node and monitoring host configuration, sensor nodes collect temperature, $\mathrm{pH}$, dissolved oxygen data is stored to the ARM processor,the ARM processor processesthe mean values for each acquisition of every 5 min,and the results will be sent to the router, which for data fusion based on adaptive weighted algorithm for multiple sensor nodes to transmit data, transmit the processed data to the coordinator through the Zig Bee network. The data fusion in sensor nodes reduces the amount of information,reduce the network burden of wireless sensor networks. The main control chip STM32F103ZET6 is connected with the monitoring terminal by GPRS, LAN and remote monitoring, the structural diagram of the system is shown in Figure 1.

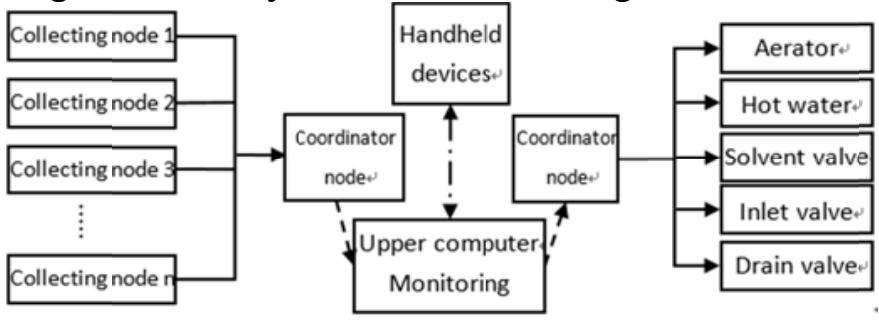

Figure 1 System overall structure 


\section{System hardware design}

\subsection{Sensor node design}

Sensor nodes mainly complete information acquisition, data processing and data transmission, the hardware platform mainly includes micro controller, communication module, sensor module and power supply module, etc.The sensor module is responsible for controlling the temperature, $\mathrm{pH}$ value and dissolved oxygen of each monitoring area. ARM processor STM32F103ZET6 is responsible for controlling the whole sensor nodes. The average value of 5 CC2530 is transmitted by radio frequency module CC2530, the average value of $5 \mathrm{~min}$ is transmitted. The power module is responsible for providing energy for each module.

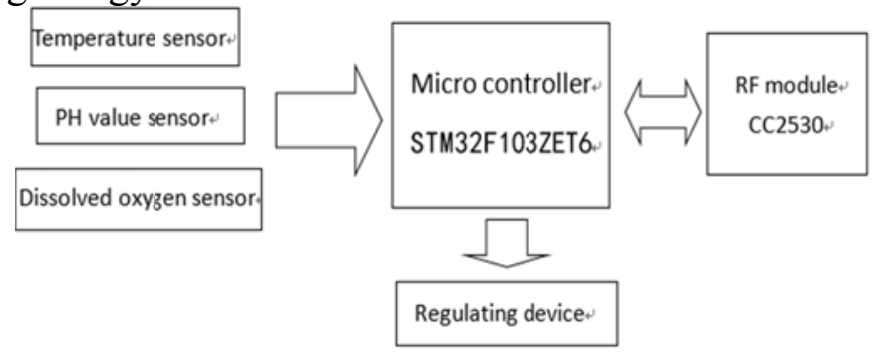

Figure 2 Sensor node structure diagram

\subsection{Routing node design}

Compared with the sensor node, the sensor module is less than the sensior module, the sensor node is sent over the data for further integration, and complete the forwarding of information, its hardware system block diagram is shown in figure 3.

\subsection{Coordinator node design}

The coordinator node is composed of STM32F103ZET6 processor CC2530, RF module SIM900 and GPRS ARM module, and the hardware system block diagram is shown in Figure 4. The coordinator node function is the information of the network construction, the network management, the transmission of the routing node through the RF module. Stm32f103zet6 processor through the RS-232 and CC2530 connection, the radio receiving information processing, and through GPRS ARM processor transmitted data conversion to standard Ethernet data format, to achieve network communication, so as to realize the monitoring of local area network and external network with PC.

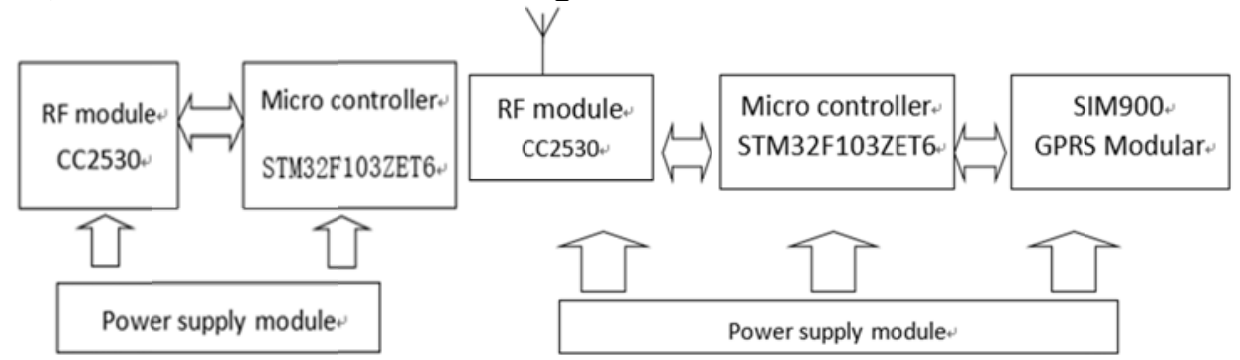

Figure 3 Routing node structure diagram Figure 4 Coordinator node structure diagram

\section{System software design}

\subsection{Sensor node software design}

Sensor node is an important part of the monitoring system. It is the source of all kinds of environmental parameters, and the program flow chart is shown in Figure 5. First of all, the initialization of ARM processor, RF module CC2530 initialization protocol stack, Bee Zig network, the sensor circuit initialization and start collecting all kinds of environmental parameters, set up 1 times per $1 \mathrm{ARM}$, min processor, min processor for each set of parameters to calculate the average value of the processed data through Bee Zig network wireless router. 


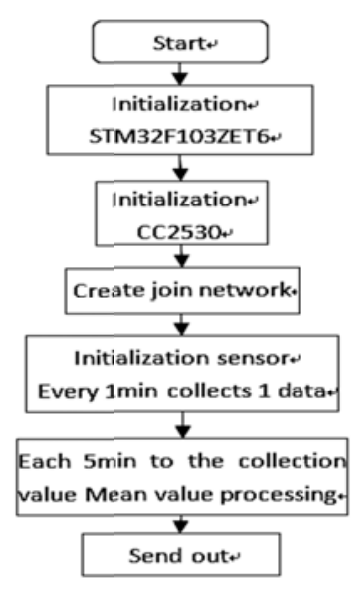

Figure 5 Sensor node flow chart

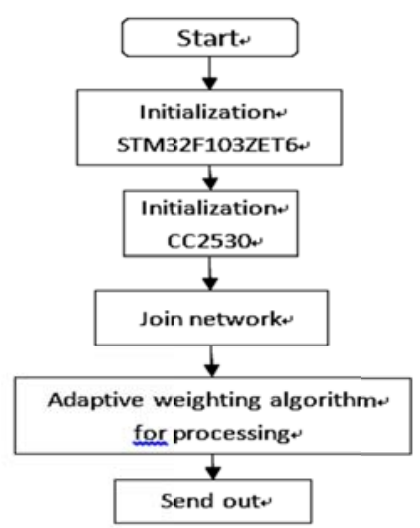

Figure 6 Router node flow chart

\subsection{Software design of router node}

The router node program flow chart is shown in figure 6. And sensor nodes are similar, each module is initialized, added to the Bee Zig network, the wireless received data is based on the adaptive weighted algorithm of information fusion, the fusion data is transmitted to the router by RF module.The basic idea of the adaptive weighted fusion algorithm is to find the optimal weighting factors for each sensor in the adaptive way, so that the $\mathrm{X}^{\wedge}$ can achieve the best results when the total mean square error is minimum. Let $\mathrm{n}$ sensor nodes send routing node received the information, the difference of sigma 12, sigma 22,... Sigma, N2; to estimate the true value of $\mathrm{X}^{\wedge}$, the measurements of sensors were $\mathrm{X} 1, \mathrm{X} 2, \ldots, \mathrm{Xn}$ they are independent of each other, and are unbiased estimates of $\mathrm{X}^{\wedge}$; the weighting factor of each sensor is $\mathrm{N} 1, \mathrm{~N} 2, \ldots, \mathrm{Nn}$, the algorithm flow is shown in figure 7.

\subsection{Software design of coordinator node}

The coordinator node program flow chart is shown in Figure 8. The ZigBee network is responsible for setting up the wireless data. If it is the GPRS module, the data is transmitted to the network. If it is to monitor the transmission of the host computer, the corresponding node is transmitted to the corresponding node.

\section{System test}

After the completion of the system design, the experimental application of the seawater aquaculture field in Qingdao: in the monitoring area, 30 sensor nodes are randomly deployed, the 5 router nodes, the 1 coordinator nodes. Sensor nodes respectively on the $\mathrm{pH}$ value, dissolved oxygen concentration, temperature of real-time monitoring, measurement information through wireless transmission to coordination node, via GPRS PC monitor, specific security set mariculture according to safety regulations. The upper computer software can set the alarm threshold, if there is more than the alarm threshold in the acquisition data, the alarm can be viewed through the history of the alarm. Figure 9 for the PC software monitoring results, as shown in Figure 9, as long as the sensor measurement data beyond the warning threshold will generate alarm.

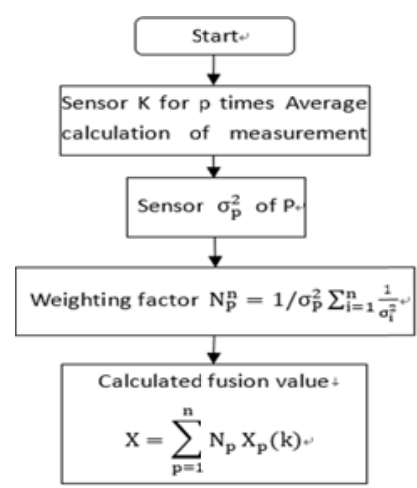

Figure 7 Adaptive weighted algorithm flow

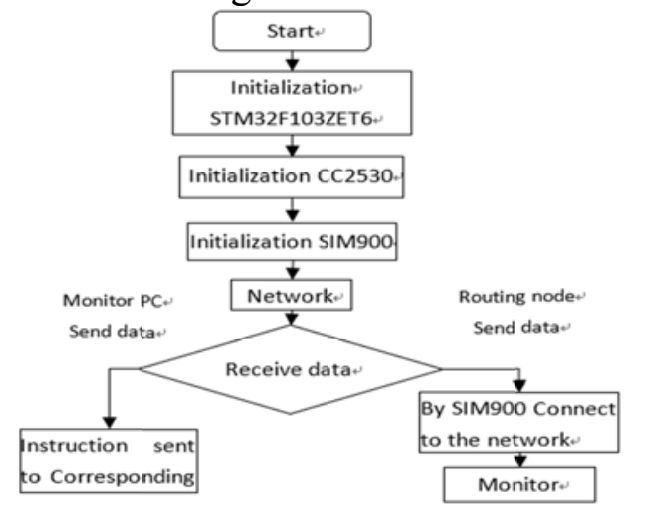

Figure 8 Coordinator node flow chart 


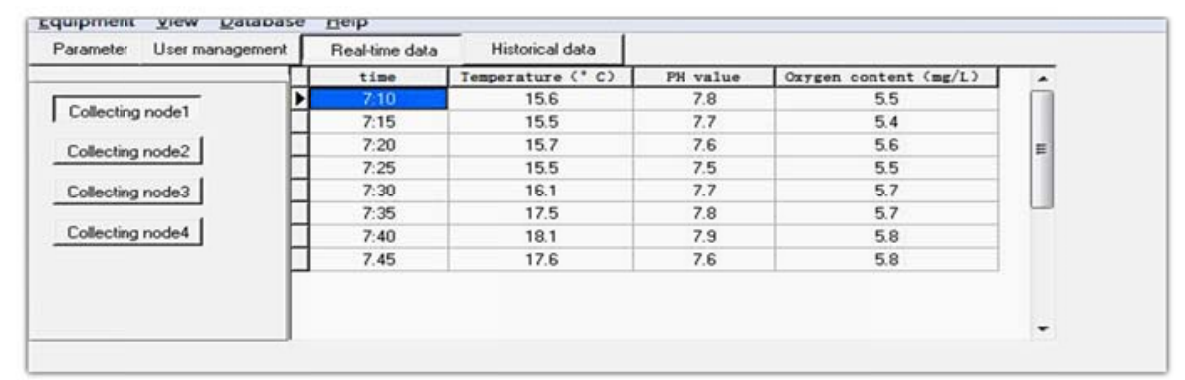

Figure 9 System monitoring results

\section{Concluding remarks}

ARM processor STM32F103ZET6, RF chip CC2530 and GPRS as the core of the design of the water aquaculture monitoring system, the expansion is convenient, can be in the shallow water area, deep water area will increase or delete sensor nodes, to avoid monitoring blind. Acquisition of the $\mathrm{pH}$ value, dissolved oxygen concentration, temperature information, in sensor nodes and routing nodes respectively by the arm processor for average and adaptive weighted algorithm of information fusion based on, the treated data by Zig Bee wireless transmit to the coordinator, the coordinator through the GPRS access network. On the PC monitor by PC software monitoring results indicate that, for data exceeds the set alarm threshold generates a warning, with strong practicability.

\section{Acknowledgements}

Research on Key Technologies of water quality monitoring in sea cucumber based on Zigbee network(J14LN82)

\section{References}

[1]Zhang Shuqing, embedded MCU STM32 design and application technology, National Defense Industry Press, May 2015.

[2] Jiang Zhong ,Liu Dan,ZigBee technology and training course - based on CC2530 wireless sensor network technology, Tsinghua University 2014-5-1.

[3]Ling Ming, Embedded system advanced C language programming, Beihang University press, January 2011.

[4]Tian Zhen, Yuan Dongfeng, Liang Quanquan. Energy efficiency analysis of error control in wireless sensor networks [J],Journal of communication, 2008,11:77-83. 